\title{
Evaluation of the relationship between neutrophil- to-lymphocyte ratio and 25-hydroxy vitamin D levels in hemodialysis patients, Isfahan, Iran
}

\author{
Azar Baradaran ${ }^{1,2}{ }^{\circledR}$, Zahra Hoseini ${ }^{*}$, Parin Hedayati ${ }^{4}$, Reyhaneh Shirvani ${ }^{3}$ \\ 'Department of Pathology, Isfahan University of Medical Sciences, Isfahan, Iran \\ ${ }^{2}$ Infectious Disease and Tropical Medicine Research Center, Isfahan University of Medical Sciences, Isfahan, Iran \\ ${ }^{3}$ Faculty of Medicine, Student Research Center, Isfahan University of Medical Sciences, Isfahan, Iran \\ ${ }^{4}$ Department of Hemodialysis, Amin Hospital, Isfahan, Iran
}

Correspondence to:

Zahra Hoseini, Email:

hoseiniz2020@yahoo.com

Received: 11 Jan. 2021

Accepted: 4 June 2021

ePublished: 3 July 2021

Keywords: Neutrophil-tolymphocyte ratio, Vitamin $\mathrm{D}$, End-stage renal disease, Hemodialysis, Inflammation, Inflammatory markers

\begin{abstract}
Introduction: Chronic low-grade inflammation is a comorbid factor in Chronic kidney disease (CKD), and especially in chronic dialysis patients. Recently, neutrophil-to-lymphocyte ratio (NLR), which is very available and affordable, has emerged as an inflammatory index in many disorders such as CKD. Several studies also have shown the anti-inflammatory effects of vitamin $D$, and scientists believe that we must prevent vitamin D deficiency in CKD patients. Vitamin D supplementation may decrease mortality and morbidity in end-stage renal disease (ESRD) patients.

Objectives: There are few studies on the association between serum 25-hydroxy vitamin D levels and NLR in ESRD patients. Therefore, we tried to evaluate this correlation, hoping to be a key for more researches. Patients and Methods: We enrolled 140 ESRD hemodialysis patients in a cross-sectional study and evaluated the correlation of NLR with serum 25-hydroxy vitamin D levels and some demographic factors in these patients.

Results: Our results showed a statistically significant negative correlation between NLR and serum 25 -hydroxy vitamin $\mathrm{D}$ levels $(P=0.010, \mathrm{r}=-0.216)$. However, other variables such as disease duration and age had no correlation with NLR $(P=0.649, \mathrm{r}=-0.039$ and $P=0.781, \mathrm{r}=-0.024$, respectively $)$. We also used a linear regression test to check any confounder associated with NLR, and the regression was only significant for serum 25-hydroxy vitamin D levels $(P=0.011, \mathrm{~B}=-0.009)$.

Conclusion: Based on the results, serum 25-hydroxy vitamin D levels can be considered a predictor for NLR in hemodialysis patients.
\end{abstract}

\section{Introduction}

Citation: Baradaran A, Hoseini Z, Hedayati $P$, Shirvani R. Evaluation of the relationship between neutrophil-to-lymphocyte ratio and 25-hydroxy vitamin $\mathrm{D}$ levels in hemodialysis patients, Isfahan, Iran. J Prev Epidemiol. 2021;6(2):e26 doi: $10.34172 /$ jpe.2021.26
The role of low-grade inflammation in the pathogenesis of many chronic diseases is an agreed issue, and the inflammatory base of many chronic diseases revealed in the last decades (1). Chronic kidney disease (CKD) is one of these diseases. It is a progressive and non-reversible disorder that is a health problem worldwide. The micro-inflammatory state of CKD leads to atherosclerosis and cardiovascular disease and an increased rate of morbidity and mortality $(2,3)$. In other words, chronic subclinical inflammation is regarded as a comorbid factor in $\mathrm{CKD}$, and especially in chronic dialysis patients (4). Recently, neutrophil-to-lymphocyte ratio (NLR) has emerged as an inflammatory marker for several chronic subclinical inflammatory diseases such as $\operatorname{CKD}(5,6)$. Some facts explain how calculating this ratio can be an indicator of inflammation. One of these

\begin{abstract}
Key point
In a study on 140 hemodialysis patients, we found a statistically significant negative correlation between NLR and serum 25-hydroxy vitamin D levels $(P=0.010, r=-0.216)$. We concluded that 25-hydroxy vitamin $\mathrm{D}$ levels might be considered a predictor for NLR in hemodialysis patients.
\end{abstract}

facts is that neutrophil has inflammatory effects (7), and its count represents the inflammation (6), while inflammation itself increases lymphocyte apoptosis (8). Besides, lymphocytes mostly have a regulatory function in the immune system (9), and their count shows the general stress and nutritional state, not inflammation (6). The increased neutrophil count with the decreased lymphocyte count predicts mortality in hemodialysis patients (10). In addition, NLR can determine the CKD progression toward the hemodialysis state (11). 
Another factor that is noteworthy in CKD is the serum level of vitamin D. Vitamin D deficiency, including the low level of serum 25-hydroxy vitamin $\mathrm{D}$ and 1,25(OH)2D, has been reported in CKD and dialysis patients generally.

Vitamin D deficiency prompts secondary hyperparathyroidism and its complications (12). An optimal level of serum 25-hydroxy vitamin D has been considered more than $30 \mathrm{ng} / \mathrm{dL}$ (13). However, studies on the optimal vitamin $\mathrm{D}$ level and the safety and effectiveness of vitamin $\mathrm{D}$ administration in these patients are continuing (14).

Vitamin D supplementation may decrease mortality and morbidity in end-stage renal disease (ESRD) patients (3). However, metastatic calcification is the dramatic result of the high level of calcium and phosphorous and can occur by using supplements $(3,15)$. Therefore, the general recommendation of taking vitamin $\mathrm{D}$ supplementation needs more researches.

A study in 2016 showed the correlation of $25(\mathrm{OH})$ D serum levels and inflammatory markers such as NLR in hemodialysis patients (16). Another cross-sectional study in 2019 on 129 maintenance hemodialysis patients revealed that serum 25-hydroxy vitamin D levels has a significant but weak inverse correlation with NLR (17).

The effect of vitamin D on inflammation and the impact of inflammation on vitamin D level are controversial issues. However, several studies show the anti-inflammatory effects of vitamin D (18-20).

\section{Objectives}

Since few studies have been carried out on the association between vitamin D and NLR in hemodialysis patients, we evaluated the correlation between NLR and serum 25-hydroxy vitamin D levels, hoping to be a key for more researches.

\section{Patients and Methods}

We conducted this cross-sectional study in 2021 in hemodialysis departments of Amin and Fatemiyeh hospitals, Isfahan, Iran. Blood sampling of hemodialysis patients routinely is conducted to check some parameters such as $\mathrm{CBC}$ and 25-hydroxy vitamin D levels. We were present in the hemodialysis departments on the days of blood sampling. After taking consent from patients and considering some inclusion and exclusion criteria, finally, 140 patients were selected. The inclusion criteria were; the ESRD patients who were at least 18 years old, had been on hemodialysis for at least three months, and had no special conditions such as drug or alcohol addiction (based on patient self-expression). The exclusion criteria based on their medical history and physical examination were; having an active infection, having malignancy or any active inflammatory disease, and taking any immunosuppressive drugs. We write information including age, gender, duration of being on hemodialysis, and having a medical history of hypertension (HTN) disease, diabetes mellitus (DM), or polycystic kidney disease. Then we followed up their blood test results to obtain NLR and the serum level of $25(\mathrm{OH}) \mathrm{D}$.

\section{Statistical analysis}

We analyzed data by using the SPSS software version 25. All quantitative variables followed the normal distribution. We also used the Pearson correlation coefficient, independent t-test, and linear regression test. The significance level in the present study was less than 0.05 .

\section{Results}

One hundred forty patients participated in this study, 89 of whom were male, and 51 were female. Ninety-five patients had DM, 79 patients had HTN disease, and no one had polycystic kidney disease. The mean age of patients was $61.9 \pm 12.9$ years. They have been on hemodialysis for an average of $2.94 \pm 2.43$ years. The mean of NLR was $2.39 \pm 0.94$, and the average serum levels of vitamin $\mathrm{D}$ was $36.03 \pm 23.30 \mathrm{ng} / \mathrm{mL}$. The maximum and minimum amount of these variables are in Table 1.

The mean level of 25-hydroxy vitamin D levels was compared in subgroups including men or women, patients with or without HTN disease, and patients with or without DM. There were no statistically significant differences between men or women $(P=0.900)$ and between patients with or without HTN disease $(P=0.368)$. However, the 25-hydroxy vitamin D serum levels were significantly different between diabetic and non-diabetic patients $(P=0.043)$. The mean of NLR also was evaluated in these subgroups, and there was no statistically significant difference (gender; $P=0.298, \mathrm{HTN} \pm ; P=0.191, \mathrm{DM} \pm$; $P=0.463$; Table 2).

We evaluated the correlation of serum levels of $25(\mathrm{OH}) \mathrm{D}$ and NLR by Pearson's correlation coefficient test. We found a statistically significant negative correlation between these two variables $(P=0.010, r=-0.216)$. However, other variables such as disease duration and age did not correlate with NLR $(P=0.649, \mathrm{r}=-0.039$ and $P=0.781, \mathrm{r}=-0.024$, respectively). We also used a linear regression test to check

Table 1. Mean and standard deviation of the basic information

\begin{tabular}{|c|c|c|c|c|}
\hline Variable & Minimum & Maximum & Mean & Standard Deviation \\
\hline Age $(y)$ & 28.00 & 85.00 & 61.9 & 12.9 \\
\hline NLR & 0.28 & 5.94 & 2.39 & 0.94 \\
\hline 25-hydroxy vitamin D levels ( $\mathrm{ng} / \mathrm{mL})$ & 8.00 & 106.00 & 36.03 & 23.30 \\
\hline Disease duration & 0.30 & 11.00 & 2.94 & 2.43 \\
\hline
\end{tabular}

NLR: Neutrophil-to-lymphocyte ratio. 
any confounder associated with NLR, and the regression was only observed for vitamin $\mathrm{D}(P=0.011, \mathrm{~B}=-0.009)$ (Tables 3 and 4).

\section{Discussion}

Two cross-sectional studies in 2016 (16) and 2019 (17) showed the negative correlation of $25(\mathrm{OH}) \mathrm{D}$ serum levels and inflammatory markers including NLR in hemodialysis patients $(P<0.001, \mathrm{r}=-0.408, \quad P=0.013, \mathrm{r}=-0.219$, respectively). Similar to these studies, we showed the existence of this correlation. This result means that higher levels of serum 25(OH)D are associated with lower ratio of NLR in hemodialysis patients. Since NLR is a subclinical inflammatory marker (21), this negative correlation may be consistent with reports about the anti-inflammatory effects of 25(OH)D levels. In our study, DM and HTN that are the two main underlying causes of ESRD (22) were not the confounders of NLR. Only 25-hydroxy vitamin D levels were meaningful in association with NLR. However, some studies have shown the association of NLR with some factors in diabetic or hypertensive patients. For instance, in DM type 2 patients NLR median has been significantly $(P<0.001)$ elevated comparing to healthy controls [2.44 (1.9) versus 1.5 (0.9), respectively] (23). In addition, in type $2 \mathrm{DM}$ patients, increased NLR levels were associated with elevated $\mathrm{HbA1c}$ and poor glycemic control (24). Furthermore, patients with DM who were vitamin $\mathrm{D}$-deficient had the highest neutrophil count $(\mathrm{P}=$

Table 2. Comparison of the serum 25-hydroxy vitamin D levels and NLR across gender, DM and HTN

\begin{tabular}{llccc}
\hline Variable & & Mean & SD & P value \\
\hline Vitamin D & Male & 36.22 & 23.85 & \multirow{2}{*}{0.900} \\
levels & Female & 35.70 & 22.54 & \\
\multirow{2}{*}{ NLR } & Male & 2.44 & 1.01 & \multirow{2}{*}{0.298} \\
& Female & 2.28 & 0.79 & \\
Vitamin D & With hypertension & 37.59 & 23.95 & \multirow{2}{*}{0.368} \\
levels & Without hypertension & 34.00 & 22.47 & \\
NLR & With hypertension & 2.48 & 1.014 & \multirow{2}{*}{0.191} \\
& Without hypertension & 2.27 & 0.83 & \\
Vitamin D & With diabetes mellitus & 33.29 & 21.49 & \multirow{2}{*}{0.043} \\
levels & Without diabetes mellitus & 41.81 & 26.05 & \\
\multirow{2}{*}{ NLR } & With diabetes mellitus & 2.43 & 0.94 & \multirow{2}{*}{0.463} \\
& Without diabetes mellitus & 2.30 & 0.93 & \\
\hline
\end{tabular}

NLR: Neutrophil-to-lymphocyte ratio.
0.001) (25). Besides, a 9-year cohort study on 6278 patients revealed a significant association between the NLR and incident HTN (26). Hence, it seems that more studies are needed, especially for evaluating underlying diseases, to accurately determine the value of NLR and its relationship with 25(OH)D in nephrology.

\section{Conclusion}

Our study showed a statistically significant negative correlation between 25-hydroxy vitamin D levels and NLR in hemodialysis patients. Thereby, serum 25-hydroxy vitamin D levels may be considered a predictor for NLR in hemodialysis patients. However, more studies are needed.

\section{Limitations of the study}

We did this study in only two departments of hemodialysis. More studies with more sample sizes are needed, especially for evaluating the effect of underlying causes of ESRD. Besides, lots of ESRD patients take vitamin D supplements. Neutrophils have a circulating half-life of 6-8 $\mathrm{h}$, and they are produced at a rate of $5 \times 10^{10}-10 \times 10^{10}$ cells/day (27). Therefore, we checked the serum level of $25(\mathrm{OH}) \mathrm{D}$ in association with NLR regardless of the source of vitamin $\mathrm{D}$ or time course of taking vitamin $\mathrm{D}$ supplementation. However, maybe more studies with controlling these factors may also be required.

\section{Authors' contribution}

The principal investigators of the study were $A B$ and $\mathrm{ZH}$. They were included in preparing the concept and design of study and writing the manuscript and critically evaluated the intellectual contents. $\mathrm{PH}$ and $\mathrm{ZH}$ participated in designing the method of study, and data collection. RS participated in data collection and drafted the manuscript. All authors have read and approved the content of the manuscript and confirmed the accuracy or integrity of any part of the work.

Conflicts of interest

The authors declare no conflict of interests.

\section{Ethical issues}

The research followed the tenets of the Declaration of Helsinki. The Ethics Committee of Isfahan University of Medical Sciences approved this study. The institutional ethical committee at Isfahan University of Medical Sciences approved the study protocols (IR. MUI.MED.REC.1399.1073). Accordingly, informed consent was

Table 3. Pearson's correlation between NLR and other effective factors

\begin{tabular}{|c|c|c|c|c|c|c|}
\hline Variable & & Disease duration & Vit D & Gender & Age & NLR \\
\hline \multirow{2}{*}{ Disease duration } & Pearson $(r)$ & 1 & 0.242 & 0.043 & 0.124 & -0.039 \\
\hline & $P$ value & - & 0.004 & 0.618 & 0.144 & 0.649 \\
\hline \multirow{2}{*}{ Vitamin D levels } & Pearson $(r)$ & 0.242 & 1 & -0.011 & -0.038 & -0.216 \\
\hline & $P$ value & 0.004 & - & 0.900 & 0.654 & 0.010 \\
\hline \multirow{2}{*}{ Gender } & Pearson ( $r$ ) & 0.043 & -0.011 & 1 & 0.001 & -0.083 \\
\hline & $P$ value & 0.618 & 0.900 & - & 0.993 & 0.330 \\
\hline \multirow{2}{*}{ Age } & Pearson $(r)$ & 0.124 & -0.038 & 0.001 & 1 & -0.024 \\
\hline & $P$ value & 0.144 & 0.654 & 0.993 & - & 0.781 \\
\hline
\end{tabular}

NLR: Neutrophil-to-lymphocyte ratio. 
Table 4. Linear regression to predict the neutrophil-to-lymphocyte ratio

\begin{tabular}{lccc}
\hline \multirow{2}{*}{ Variable } & \multicolumn{2}{c}{ Unstandardized coefficients } & \multirow{2}{*}{$\boldsymbol{P}$ value } \\
\cline { 2 - 3 } & Beta coefficient & Standard error & \\
\hline Diabetes mellitus & 0.213 & 0.186 & 0.255 \\
Hypertension & 0.323 & 0.168 & 0.057 \\
Disease duration & 0.017 & 0.034 & 0.614 \\
Vitamin D levels & -0.009 & 0.004 & 0.011 \\
Gender & -0.232 & 0.166 & 0.166 \\
Age & -0.004 & 0.006 & 0.565 \\
\hline
\end{tabular}

taken from all participants. This study was extracted from the M.D., thesis of Zahra Hoseini at this university (Thesis\# 399971). Moreover, ethical issues (including plagiarism, data fabrication, double publication) have been completely observed by the authors.

\section{Funding/Support}

None.

\section{References}

1. Minihane AM, Vinoy S, Russell WR, Baka A, Roche HM, Tuohy $\mathrm{KM}$, et al. Low-grade inflammation, diet composition and health: current research evidence and its translation. $\mathrm{Br} J$ Nutr. 2015;114:999-1012. doi: 10.1017/S0007114515002093.

2. Jean G, Charra B, Chazot C. Vitamin D deficiency and associated factors in hemodialysis patients. J Ren Nutr. 2008;18:395-9. doi: 10.1053/j.jrn.2008.04.003.

3. Sterling KA, Eftekhari P, Girndt M, Kimmel PL, Raj DS. The immunoregulatory function of vitamin $\mathrm{D}$ : implications in chronic kidney disease. Nat Rev Nephrol. 2012;8:403-12. doi: 10.1038/nrneph.2012.93.

4. Nowak KL, Chonchol M. Does inflammation affect outcomes in dialysis patients? Semin Dial. 2018;31:388-397. doi: 10.1111/sdi.12686.

5. Yuan Q, Wang J, Peng Z, Zhou Q, Xiao X, XieY, et al. Neutrophilto-lymphocyte ratio and incident end-stage renal disease in Chinese patients with chronic kidney disease: results from the Chinese Cohort Study of Chronic Kidney Disease (C-STRIDE). J TransI Med. 2019;17:1-8.

6. S Solak Y, Yilmaz MI, Sonmez A, Saglam M, Cakir E, Unal HU, et al. Neutrophil to lymphocyte ratio independently predicts cardiovascular events in patients with chronic kidney disease. Clin Exp Nephrol. 2013;17:532-40. doi: 10.1007/s10157-0120728-x.

7. Soehnlein O, Steffens S, Hidalgo A, Weber C. Neutrophils as protagonists and targets in chronic inflammation. Nat Rev Immunol. 2017;17:248-261. doi: 10.1038/nri.2017.10.

8. Hotchkiss RS, Karl IE. The pathophysiology and treatment of sepsis. N Engl J Med. 2003:348:138-50. doi: 10.1056/ NEJMra021333.

9. Azab B, Zaher M, Weiserbs KF, Torbey E, Lacossiere K, Gaddam S, et al. Usefulness of neutrophil to lymphocyte ratio in predicting short- and long-term mortality after non-STelevation myocardial infarction. Am J Cardiol. 2010;106:4706. doi: 10.1016/j.amjcard.2010.03.062.

10. Reddan DN, Klassen PS, Szczech LA, Coladonato JA, O'Shea $\mathrm{S}$, Owen WF Jr,et al. White blood cells as a novel mortality predictor in haemodialysis patients. Nephrol Dial Transplant. 2003;18:1167-73. doi: 10.1093/ndt/gfg066.

11. Kocyigit I, Eroglu E, Unal A, Sipahioglu MH, Tokgoz B, Oymak $\mathrm{O}$, et al. Role of neutrophil/lymphocyte ratio in prediction of disease progression in patients with stage- 4 chronic kidney disease. J Nephrol. 2013;26:358-65. doi: 10.5301/jn.5000152.

12. Jean G, Souberbielle JC, Chazot C. Vitamin D in Chronic Kidney Disease and Dialysis Patients. Nutrients. 2017 Mar 25;9(4):328. doi: 10.3390/nu9040328.
13. Wang AY, Lam CW, Sanderson JE, Wang M, Chan IH, Lui SF, et al. Serum 25-hydroxyvitamin D status and cardiovascular outcomes in chronic peritoneal dialysis patients: a $3-y$ prospective cohort study. Am J Clin Nutr. 2008;87:1631-8. doi: 10.1093/ajcn/87.6.1631.

14. Saab G, Young DO, Gincherman Y, Giles K, Norwood K, Coyne DW. Prevalence of vitamin D deficiency and the safety and effectiveness of monthly ergocalciferol in hemodialysis patients. Nephron Clin Pract. 2007;105:c132-8. doi: 10.1159/000098645.

15. Fortier C, Mac-Way F, De Serres SA, Marquis K, Douville $P$, Desmeules $S$, et al. Active vitamin $D$ and accelerated progression of aortic stiffness in hemodialysis patients: a longitudinal observational study. Am J Hypertens. 2014;27:1346-54. doi: 10.1093/ajh/hpu057.

16. Mirchi E, Saghafi H, Gharehbeglou M, Aghaali M, Rezaian Z, Ghaviahd M. Association Between 25-Hydroxyvitamin D Level and Inflammatory and Nutritional Factors in Hemodialysis and Peritoneal dialysis Patients in Qom, Iran. Iran J Kidney Dis. 2016;10:205-12.

17. Kara AV, Soylu YE. The relationship between vitamin D and inflammatory markers in maintenance hemodialysis patients. Int Urol Nephrol. 2019;51:1659-1665. doi: 10.1007/s11255019-02250-x.

18. Feng X, Lv C, Wang F, Gan K, Zhang M, Tan W. Modulatory effect of 1,25-dihydroxyvitamin D 3 on IL1 $\beta$-induced RANKL, OPG, TNF $\alpha$, and IL-6 expression in human rheumatoid synoviocyte MH7A. Clin Dev Immunol. 2013;2013:160123. doi: 10.1155/2013/160123.

19. Guillot X, Semerano L, Saidenberg-Kermanac'h N, Falgarone G, Boissier MC. Vitamin D and inflammation. Joint Bone Spine. 2010;77:552-7. doi: 10.1016/j.jbspin.2010.09.018.

20. Haddad Kashani H, Seyed Hosseini E, Nikzad H, Soleimani A, Soleimani M, Tamadon MR, et al. The Effects of Vitamin D Supplementation on Signaling Pathway of Inflammation and Oxidative Stress in Diabetic Hemodialysis: A Randomized, Double-Blind, Placebo-Controlled Trial. Front Pharmacol. 2018;9:50. doi: 10.3389/fphar.2018.00050.

21. Wang X, Zhang G, Jiang X, Zhu H, Lu Z, Xu L. Neutrophil to lymphocyte ratio in relation to risk of all-cause mortality and cardiovascular events among patients undergoing angiography or cardiac revascularization: a meta-analysis of observational studies. Atherosclerosis. 2014;234:206-13. doi: 10.1016/j. atherosclerosis.2014.03.003.

22. Lea JP, Nicholas SB. Diabetes mellitus and hypertension: key risk factors for kidney disease. J Natl Med Assoc. 2002;94:7S-15S

23. Duman TT, Aktas G, Atak BM, Kocak MZ, Erkus E, Savli H. Neutrophil to lymphocyte ratio as an indicative of diabetic control level in type 2 diabetes mellitus. Afr Health Sci. 2019;19:1602-1606. doi: 10.4314/ahs.v19i1.35.

24. Hussain M, Babar MZM, Akhtar L, Hussain MS. Neutrophil lymphocyte ratio (NLR): A well assessment tool of glycemic control in type 2 diabetic patients. PakJ Med Sci. 2017;33:13661370. doi: 10.12669/pjms.336.12900.

25. Burrows NR, Hora I, Geiss LS, Gregg EW, Albright A. Incidence of End-Stage Renal Disease Attributed to Diabetes Among Persons with Diagnosed Diabetes - United States and Puerto Rico, 2000-2014. MMWR Morb Mortal Wkly Rep. 2017;66:1165-1170. doi: 10.15585/mmwr.mm6643a2.

26. Jhuang YH, Kao TW, Peng TC, Chen WL, Li YW, Chang PK, et al. Neutrophil to lymphocyte ratio as predictor for incident hypertension: a 9-year cohort study in Taiwan. Hypertens Res. 2019;42(8):1209-1214. doi: 10.1038/s41440-019-0245-3.

27. Summers C, Rankin SM, Condliffe AM, Singh N, Peters AM, Chilvers ER. Neutrophil kinetics in health and disease. Trends Immunol. 2010;31:318-24. doi: 10.1016/j.it.2010.05.006. 